\title{
La enseñanza digital en serio y el derecho a la educación en tiempos del coronavirus ${ }^{1}$
}

\author{
Digital education taken seriously and the right to \\ education in times of the coronavirus
}

\author{
Lorenzo Cotino Hueso \\ Catedrático de Derecho Constitucional \\ Departamento de Derecho Constitucional, Ciencia Política y de la Administración. \\ Facultad de Derecho, Universitat de València \\ Investigador de la Universidad Católica de Colombia \\ E-mail: cotino@uv.es
}

\begin{abstract}
Resumen: Especialmente desde una perspectiva jurídica, el estudio analiza las medidas en el ámbito educativo adoptadas en a nivel mundial y en España en razón del coronavirus: la "suspensión sin suspensión" de la educación especialmente a través de la enseñanza digital. Sin embargo, la educación digital se ha considerado "inferior", "excepcional" y sólo "complementaria" de la presencial y fuente de graves barreras y peligros para la educación. Así ha sido en el ámbito internacional y también para el TEDH, el TC y nuestra legislación. Se tiene en cuenta el especial peligro de la desigualdad en la educación digital y, ya en el caso de la pandemia, se describen algunas medidas especiales de equidad e igualdad. Sin perjuicio de este panorama más negativo, se apuesta por ver el vaso medio lleno y por hacer de la necesidad virtud. Se considera así que el coronavirus supone una oportunidad para dejar atrás una educación obsoleta del siglo XX. Se recuerda la necesidad del cambio hacia competencias digitales y el pensamiento computacional. Finalmente se hace un recorrido por los contenidos y objetivos del derecho a la educación y especialmente las obligaciones prestacionales que implica para su proyección ante los retos generales de la educación digital y la
\end{abstract}

\footnotetext{
${ }^{1}$ El presente estudio es resultado de investigación del proyecto "Derecho y Big Data", Grupo de Investigación en Derecho Público y TIC como investigador de la Universidad Católica de Colombia. De igual modo, en el marco del proyecto "La regulación de la transformación digital y la economía colaborativa" PROMETEO/2017/064 y MICINN Retos "Derechos y garantías frente a las decisiones automatizadas... (RTI2018-097172-B-C21, pendiente), al igual que de la Estancia de investigación ayuda Generalitat (AEST/2019/013).
} 
situación de la pandemia. Además de las obligaciones del Estado, se recuerdan los deberes de los padres en la educación, y también ante la educación digital en casa en razón de las circunstancias. Se concluye que la educación digital es un salvavidas en una situación como la pandemia para garantizar unos mínimos de los objetivos que garantiza el derecho a la educación. Pero es más, la pesadilla del coronavirus nos despierta de cierto letargo en la educación y nos obliga a asumir el cambio de siglo y nos brinda la oportunidad de mejorar mucho la educación gracias a la transformación digital.

Palabras clave: Derecho a la educación, internet, transformación digital, coronavirus, derecho constitucional.

Abstract: Especially from a legal perspective, the study analyzes the measures in the educational field adopted worldwide and in Spain due to the coronavirus; the "suspension without suspension" of education especially through digital teaching. However, digital education has been considered "inferior", "exceptional" and only "complementary" to face-to-face teaching and a source of serious barriers and dangers for. Thus, it has been at the international level and also for the ECHR, the TC and our legislation. The special danger of inequality in digital education is also taken into account and already in the case of the pandemic, some special measures of equity and equality are described. Notwithstanding this more negative scenario, the author prefers to see the glass half full and make a virtue of need. Then, the coronavirus is seen as an opportunity to leave behind an obsolete education of the 20th century. The need for a shift towards digital skills and computational thinking is recalled. Finally, are described the contents and objectives of the right to education and especially the obligations to provide it in the face of the general challenges of digital education and the situation of the pandemic. In addition to the obligations of the State, parents' duties in education are remembered, and also in the face of digital education at home due to circumstances. It is concluded that digital education is a life buoy in the pandemic situation to ensure a minimum of the objectives that guarantee the right to education. It is concluded that the coronavirus nightmare awakens us from some lethargy in education. It forces us to assume the turn of the century and provides us with the opportunity to greatly improve education thanks to the digital transformation. 
Keywords: Right to education, internet, digital transformation, coronavirus, constitutional law.

\section{I.- La "suspensión sin parar la escuela" a través de la enseñanza digital y los efectos negativos de suspensiones de la enseñanza}

A final de marzo de 2020 y según la UNESCO en el mundo hay más de mil quinientos millones de estudiantes afectados por el Coronavirus, lo que supone un 89,4\% del total de estudiantes inscritos en 184 países que han implantado cierres (UNESCO 2020 a). Otros países han hecho cierres localizados y en la mayor parte de países se experimentarán interrupciones educativas ${ }^{2}$. UNICEF (2020) prontamente lanzó lineamientos generales para los centros, que en líneas generales siguen manteniéndose, a saber: sigue las recomendaciones de la administración y adáptalas a tu contexto; cuenta con la comunidad educativa, fortaleciendo el clima escolar; una adecuada comunicación de crisis contiene la difusión de rumores y clarifica cuáles son las fuentes de información confiables mientras dure la situación; pon freno a la discriminación de comunidades estigmatizadas, como fueron los ciudadanos chinos; aprovecha para mejorar, la situación permite detectar antiguos problemas y mejorarlos de cara al futuro. Soluciones innovadoras a través de la educación a distancia, con una plantificación adecuada a la edad, estatus de familias o capacidad del profesorado; y transmite calma, se recuerda que en las situaciones complicadas es donde más se pone a prueba esta capacidad de impacto que tienen las instituciones educativas.

Como describen Chang y Yano (2020) ante la pandemia global las acciones mundiales están convergiendo. Más allá de las medidas propiamente sanitarias para el ámbito educativo, los países se han centrado en garantizar la continuación del aprendizaje, evitando en lo posible la interrupción. Se han tomado medidas como la introducción o ampliación de las modalidades existentes de educación a distancia, proporcionar plataformas en línea, estimular a maestros y administradores escolares a utilizar aplicaciones, generar y difundir contenidos educativos a través de la televisión y otros medios, emplear las aplicaciones ya existentes de comunicación profesores, familias y

\footnotetext{
${ }^{2}$ Los datos actualizados por países en:https://en.unesco.org/sites/default/files/covid_impact_education.csv
} 
alumnos y campañas de sensibilización o estrategias de comunicación sobre educación a distancia.

Y más allá de la educación a distancia las medidas se centran en ajustar, reprogramar y diseñar el calendario escolar y de exámenes escuela y el calendario de exámenes ha sido una prioridad; en algunos casos, incluir exámenes en línea si no se pueden cambiar las fechas (incluso se ha llegado a producir la cancelación de todas las pruebas (Florida y Washington en EEUU); programas y calendarios de capacitación de maestros y licencias de maestros; y en ayuda de las familias se brinda apoyo pedagógico en línea a los padres con materiales específicos o incluso con cursos en línea sobre cómo gestionar la relación con los alumnos (Italia) o herramientas colaborativas para compartir conocimientos y experiencias. También se tiene en cuenta que hay que afrontar el aislamiento social de los niños y se ofrece asistencia psicológica a los necesitados incluso con líneas de 24 horas y seguimientos.

Cabe tener en cuenta la experiencia anticipada de China. El Ministerio de educación el 31 de enero 2020 lanzó la política "suspender las clases sin parar la escuela"33 y, en la Universidad, "Suspensión sin suspensión"4. Todo esencialmente a través de la enseñanza digital. Se reaccionó muy rápido ante la pronta suspensión de clases presenciales y ya el 2 de febrero 22 plataformas digitales comenzaron a ofrecer más de 24.000 cursos en línea, incluidos 401 cursos experimentales de simulación virtual (CABROL, 2020).

La UNESCO rápidamente lanzó un completo listado de sesenta herramientas tecnológicas y plataformas para la educación (UNESCO, 2020 b) con sistemas de gestión de aprendizaje digital ${ }^{5}$; sistemas diseñados específicamente para teléfonos móviles ${ }^{6}$; sistemas con una fuerte funcionalidad fuera de línea ${ }^{7}$; plataformas masivas de curso abierto en línea (MOOC) $)^{8}$; contenido de aprendizaje autodirigido9; aplicaciones

\footnotetext{
${ }^{3}$ http://www.moe.gov.cn/jyb_xwfb/gzdt_gzdt/s5987/202001/t20200129 416993.html

4 http://www.moe.gov.cn/jyb_xwfb/xw zt/moe 357/jyzt_2020n/2020_zt03/zydt/zydt_gxdt/tkbtx/

5 Pizarra, CenturyTech, ClassDojo, Edmodo, Edraak, EkStep, Google Classroom, Moodle, Nafham, Schoology, Seesaw, Skooler, Study Sapuri.

${ }^{6}$ Cell-Ed, Eneza, Funzi, KaiOS, Ubongo, Ustad Mobile.

${ }^{7}$ Can't wait to Learn, Kolibri, Ustad Mobile.

${ }^{8}$ Alison, Canvas, Coursera, EdX, University of the People, Icourses, Future Learn.

9 Byju's, Discovery Education, Geekie, Khan Academy, KitKit School, LabXchange, Mindspark, Mosoteach, OneCourse, Quizlet, Siyavula, YouTube.
} 
de lectura móvil ${ }^{10}$; plataformas de colaboración que admiten la comunicación de video en vivo ${ }^{11}$; y herramientas para crear contenido de aprendizaje digital ${ }^{12}$.

Desde esta institución se ha creado la World Coalition for Education ${ }^{13}$ que incluye a las Agencias de las Naciones Unidas, las organizaciones internacionales, el sector privado y los representantes de la sociedad civil para una respuesta educativa a nivel mundial, regional y nacional. Ya en los primeros días, los miembros del sector privado incluyen a los grandes del sector tecnológico (GSMA, Microsoft, Weidong, Google, Facebook, Amazon, Coursera, Zoom). Del tercer sector Khan Academy, Profuturo, Associated Press, BBC World Service, Global Book Alliance, INEE. Otros se han ido sumando.

Sanz (2020) ha recordado la literatura sobre efectos negativos de la suspensión de clases. Así, a partir de experiencias por cierres por nevadas, se indica que los efectos pueden mitigarse si hay una respuesta coordinada y no se alarga en el tiempo (GOODMAN 2014). Más preocupante son los resultados internacionales de Jaume y Willén (2019) en sus estudios sobre suspensiones de educación por periodos más prolongados.

A partir del análisis de la experiencia Argentina, con 1.500 huelgas de profesorado educación entre 1983 y 2014, en algunos rangos de edad, los estudiantes perdieron 88 días de escuela primaria debido a las huelgas. Pues bien, se indica que hubo importantes efectos para los mismos en el mercado laboral de quienes lo padecieron. Así, las ganancias laborales se redujeron para hombres y mujeres en 3.2 y $1.9 \%$. De igual modo se dio un aumento en el desempleo por disminución en habilidades. Especialmente se indica que los efectos en los primeros grados son más grandes que en los últimos grados. De hecho se señala que los efectos tuvieron reflejo en los hijos de los que padecieron las huelgas. Considero que sin duda ha de haber efectos negativos y que pueden dejar huella en el tiempo. No obstante, cabe señalar que actualmente hay grandes posibilidades de educación a distancia y un profesorado e instituciones dedicados a intentar garantizarla.

\footnotetext{
${ }^{10}$ African Storybook, Global Digital, LibrarLezioni sul sofà, StoryWeaver, Worldreader.

${ }^{11}$ Dingtalk, Lark, Hangouts Meet, Teams, Skype, Zoom.

${ }^{12}$ Thinglink, Buncee, EdPuzzle, Kaltura, Nearpod, Pear Deck, Squigl.

${ }^{13}$ https://en.unesco.org/covid19/\%20educationglobalcoalition

https://es.unesco.org/covid19/globaleducationcoalition
} 


\section{II.- En España, enseñanza online, "mientras dure la guerra" y otras medidas}

En España, tras una inicial quietud, el 11 de marzo 2020 el Consejo de Ministros, "recomendó" a las Comunidades Autónomas suspender las clases. La decisión ya se había adoptado en zonas del País Vasco, Madrid y la Rioja algo antes y se fueron sumando en cadena las distintas Comunidades. En todo caso, el Real Decreto 463/2020, de 14 de marzo de alarma en su artículo 9 reguló "Medidas de contención en el ámbito educativo y de la formación”. Así: 10 "se suspende la actividad educativa presencial en todos los centros y etapas, ciclos, grados, cursos y niveles de enseñanza" contemplados en el artículo 3 de la Ley Orgánica 2/2006, de 3 de mayo, de Educación, incluida la enseñanza universitaria, así como cualesquiera otras actividades educativas o de formación impartidas en otros centros públicos o privados." $\left(2^{\circ}\right)$ "Durante el período de suspensión se mantendrán las actividades educativas a través de las modalidades a distancia y «on line», siempre que resulte posible." Como analizo en detalle en otros estudios (Cotino 2020 a y b), la regulación del estado de alarma implica un desplazamiento de la normativa ordinaria. Así, este artículo 9 del decreto de alarma implica una modulación del ejercicio del derecho a la educación que bien puede considerarse una restricción del mismo. Sin embargo, no puede considerarse que la suspensión de clases presenciales implique una suspensión material del derecho. Nuestra Constitución no prevé la suspensión del derecho a la educación en su artículo $55 \mathrm{CE}$, ni está prevista una medida específica respecto de la educación en la Ley Orgánica 4/1981, de 1 de junio.

Se seguía en buena medida la estela de Italia. Ahí, artículo $1.1^{\circ} \mathrm{h}$ ) del Decreto del Presidente del Consejo de Ministros de 8 de marzo dispuso que "se suspenden los servicios educativos [...] y actividades didácticas en escuelas de todos los niveles, así como la frecuencia de actividades escolares $\mathrm{y}$ formativas superior, incluidas universidades e instituciones [...] se detiene en cualquier caso la posibilidad de realizar actividades de capacitación remota que excluyen cursos para médicos en formación especializada y cursos de formación específicos en medicina general, así como las actividades de los alumnos de la profesiones de la salud. Los órganos de gestión aseguran la limpieza del entornos y requisitos administrativos y contables relacionados con los servicios de cuidado de niños referidos, no parte de círculos didácticos o instituciones integrales." 
El Ministerio de Educación lanzó el portal Aprendo en casa para canalizar recursos, herramientas y aplicaciones educativas de calidad a disposición del profesorado, de las familias y del propio alumnado ${ }^{14}$, que integra también materiales y recursos puestos en marcha por las Comunidades Autónomas, entidades privadas y otros agentes a los que agradecemos su cooperación. Se estructuran en recursos, formación y apoyo, otras iniciativas, "te ayudamos" y "familias", también con apoyo de programación especial en TVE. Todas las Comunidades Autónomas han lanzado webs o secciones sobre normativa, recursos, aplicaciones ante el cese temporal de la actividad lectiva ${ }^{15}$.

Por cuanto a otras medidas relevantes, en Conferencia sectorial ${ }^{16}$ de 25 de marzo de 2020 se acordaron cambios en el calendario y en las pruebas de acceso a la Universidad (EBAU) y el "modelo de examen se ajustará a la excepcionalidad que supone el actual estado de alarma para no perjudicar al alumnado". Al respecto, el Consejo Escolar del Estado el 30 de marzo $2020^{17}$ parece proponer que se den "los mismos criterios generales en todas las comunidades, para evitar posibles discriminaciones". La Conferencia sectorial también acordó flexibilizar las prácticas de Formación Profesional y el número mínimo de 220 horas de prácticas, así como la suspensión de las pruebas de evaluación diagnóstica de $3^{\circ}$ y $6^{\circ}$ de primaria y $4^{\circ}$ de la ESO. Otras medidas particulares se han adoptado, como en el ámbito de la formación militar (Orden DEF/291/2020, de 26 de marzo).

También, desde el Consejo Escolar del Estado al parecer se propuso la finalización del curso por medios telemáticos y una importante relajación de requisitos académicos, como la exigencia de superación de la última evaluación. Ello al tiempo de no avanzar en los contenidos, sino esencialmente repasar los de las evaluaciones anteriores.

A estas medidas hay que añadir las adoptadas concretamente en cada comunidad autónoma, como las relativas a que se reciba la cuantía de becas para comedor; no abono de cuotas de comedor; suspensión de todas las evaluaciones de diagnóstico; acceso a plataformas y contenidos; suspensión de las prácticas en centros de trabajo; teletrabajo del personal laboral educativo, así como la suspensión de procedimientos administrativos, con especial incidencia respecto de admisiones a centros, solicitud de admisiones, traslados, conciertos educativos y cuestiones de personal.

\footnotetext{
${ }^{14} \mathrm{https} / / /$ aprendoencasa.educacion.es/

$15 \mathrm{https} / / /$ aprendoencasa.educacion.es/comunidades-autonomas/

${ }^{16} \mathrm{https} / / / \mathrm{www} . e d u c a c i o n y f p . g o b . e s /$ prensa/actualidad/2020/03/20200325-sectorial.html

17 https://www.elmundo.es/espana/2020/03/31/5e833688fc6c8374328b459f.html
} 
Se suscitaron dudas sobre pago de cuotas de colegios concertados y privados y a falta de decisiones normativas el criterio que se afirma es que se siguen prestando en la medida de lo posible los servicios educativos por lo que parece justificado el cobro respecto de centros concertados. No así respecto de servicios de guardería o academias privadas ${ }^{18}$, existiendo plataformas de reclamación por consumidores ${ }^{19}$.

\section{III.- El vaso medio vacío. La educación digital es "inferior" y solo complementaria}

\section{1.- Una visión internacional apocalíptica del "tsunami digital" en educación}

Altas instituciones como el Relator de educación de la ONU (NACIONES UNIDAS 2016) han percibido de manera muy conservadora y casi apocalíptica el "tsunami digital" para la educación ( $n^{\circ}$ 94). Pese a que se trate de una percepción quizá conservadora y excesivamente negativa, sin duda señala las guías de hacia dónde hay que concentrar todos los esfuerzos y medidas correctoras. Así, además de los graves riesgos para la igualdad que luego se refieren, se señalan los siguientes riesgos:

- "La utilización de las tecnologías digitales entraña el riesgo de que se vean socavados los valores humanos en la educación, así como la calidad de esta, especialmente en lo que respecta a los títulos y diplomas que se expiden de manera fraudulenta." ( $\left.n^{\circ} 110\right)$.

- "menoscaba[n] la capacidad de los estudiantes de contemplar y pensar de manera crítica, acabar con los valores humanos en la educación y allanar el camino para la comercialización de la educación.”

- Se aprecian graves problemas de "calidad y problemas relativos al reconocimiento de los títulos y diplomas" (n 48 y ss.). Así, "los métodos de enseñanza han sido criticados" $\left(n^{\circ} 51\right)$, "es preocupante la nueva tendencia de cualificar mediante cursos en línea masivos y abiertos de aprendizaje a distancia" ( $\mathrm{n}^{\circ}$ 52) y "existe el riesgo de fraude relacionado con la expedición de títulos en línea" ( $\left.n^{\circ} 53\right)$.

- Además, los “índices de finalización sumamente bajos” (nº 58).

- Por cuanto a los docentes (VII. no 55 y ss.) "La autonomía de los maestros para enseñar de la manera que, a su juicio, es la mejor puede verse socavada por los modelos

18 https://www.rtve.es/noticias/20200327/tengo-seguir-pagando-cuota-guarderia-colegio-duranteconfinamiento/2010927.shtml

${ }^{19}$ https://www.facua.org/Covid-19 
de educación basados en la tecnología [y...] se está viendo menoscabada la capacidad de los maestros para seleccionar el contenido que consideran pertinente para sus condiciones y clases locales."

- "La educación debe impartirse adaptándose a las necesidades de los estudiantes y al contexto local", pero en la enseñanza online el "método abrumadoramente occidental y anglo-estadounidense", lo cual es contrario a la libertad académica (nº 56 y 57 ).

- Las plataformas se centran "más en la difusión de contenidos y bastante menos en la participación e interacción del educando" (nº 60).

- Se dedica un capítulo a "Las tecnologías digitales y las fuerzas de la privatización en la educación" (XII). "Los cursos en línea masivos y abiertos y otros formatos de educación a distancia pueden promover la privatización, reducir la financiación pública y aumentar el control del equipo directivo sobre el personal académico" ( $\mathrm{n}^{\circ}$ 90). Especialmente "en las universidades a actitudes más orientadas al consumidor, y a la comercialización de los conocimiento" ( $\left.\mathrm{n}^{\circ} 89\right)$.

En conclusión, la educación presencial es superior y la digital solo puede ser complementaria: "A la luz del auge del aprendizaje en línea y basado en la web, el Relator Especial considera importante reconocer los límites del valor pedagógico de la educación a distancia y basada en la tecnología, estableciendo la superioridad del aprendizaje presencial y las interacciones humanas en la educación. Todas las formas de la educación en línea pueden ayudar a mejorar el acceso a la educación superior, pero solo si son un complemento, y no un sustituto, de las prácticas pedagógicas consagradas." ( $\left.n^{\circ} 58\right)$.

Sin duda que estas barreras y peligros hay que tenerlos en cuenta y corregirlos en la medida de lo posible. No obstante, como señalé en 2012 (COTINO, 2012), "hoy por hoy se afirma con buen sentido que las tecnologías no pueden sustituir a la buena escuela ni al buen docente, que la educación es mucho más que información y comunicación. Ni los textos escolares antes, ni las computadoras hoy, sustituyen el contacto, la interacción humana y el afecto que son base esencial del aprendizaje, especialmente de niños y jóvenes." Añadía entonces que puede llegar pronto el día en que sean los ricos quienes accedan a los buenos maestros y maestras, y los pobres quienes deban contentarse con manipular computadoras y máquinas de todo tipo. Ello se 
ha ido confirmando. Así, "Los tecnólogos de Silicon Valley no quieren que sus hijos usen los dispositivos que ellos fabrican" (BOWLES, 2018), "Los gurús digitales crían a sus hijos sin pantallas" (GUIMÓN, 2019).

Ahora bien, como también apunté en 2012, estábamos solo al principio de un camino, "las carencias que se achacan a las TIC están superándose en muy buena medida y el Derecho habrá de abrirse a las posibles demandas sociales de educación a distancia y formas semipresenciales que puedan darse en la sociedad, convergiendo, en su caso con las ya analizadas demandas de educación en casa."

\section{2.- La educación digital es inferior y excepcional para el TEDH, el TC y nuestra legislación}

Los tribunales han admitido la superioridad de la educación presencial, al menos para la educación no universitaria. Así lo han hecho en razón de la discusión constitucional sobre el homeschooling o educación en casa (COTINO, 2011, 2012). Así, la STEDH de 11 de septiembre de 2006, caso Konrad de Fritz y otros admitió la imposición de la escolarización forzosa frente a la libertad de los padres sobre la base del logro del objeto de la educación, en especial la necesaria sociabilidad del alumno. Además recordó que los padres siguen contando con las horas no lectivas para educar a sus hijos. En España, el Tribunal Constitucional rehuyó afrontar directamente la cuestión en la STC 260/1994, de 3 de octubre, caso secta "Niños de Dios". Sin embargo, la STC 133/2010, de 2 de diciembre dejó la cuestión relativamente clara. El legislador "puede optar en ejercicio del margen de libre apreciación política que le corresponde en virtud del principio de pluralismo político" (FJ $7^{\circ}$ ). Y el legislador ha optado por la escolarización obligatoria sin que ello lesione el derecho a la educación. De hecho, esta opción del legislador responde a mandatos constitucionales de artículo 27. $5^{\circ}$ (programación) y $8^{\circ} \mathrm{CE}$ (homologación). En sentido paralelo al TEDH, la finalidad del artículo 27. $2^{\circ} \mathrm{CE}$ "se ve satisfecha más eficazmente mediante un modelo de enseñanza básica en el que el contacto con la sociedad plural y con los diversos y heterogéneos elementos que la integran, lejos de tener lugar de manera puramente ocasional y fragmentaria, forma parte de la experiencia cotidiana que facilita la escolarización." Y por cuanto la proporcionalidad estricta de la medida, en la línea del TEDH se recuerda que los padres pueden educar fuera del horario escolar y que si quieren pueden fundar un centro docente $\left(\mathrm{FJ} 8^{\circ}\right)$. 
En nuestra legislación y desde antiguo, a la enseñanza no universitaria a distancia se la ha considerado como la "heredera de la enseñanza libre" (CÁMARA y PARADA, 1988: 77) y, en consecuencia no está permitida en España. Ello es así a diferencia de la educación universitaria, pues la Ley Orgánica 6/2001, de 21 de diciembre, de Universidades permite a las mismas determinar el modo de enseñanza presencial, semipresencial o a distancia en su artículo 4. $3^{\circ}$. Sin perjuicio de que se reconozca en la normativa la importancia de las competencias digitales, la enseñanza no presencial y no universitaria es completamente residual y subsidiaria en la LOE. Así, la educación no presencial queda reservada para quienes no pueden asistir, art. 3. $9^{\circ} \mathrm{LOE}$; cuando la oferta de materias se limite por razones organizativas, art. $34.5^{\circ} \mathrm{LOE}$; posible en la enseñanza de idiomas, art. 60. $3^{\circ}$ LOE o en la oferta a personas adultas, arts. 67. $1^{\circ}$ y 69. $3^{\circ} \mathrm{LOE}$.

La legislación autonómica en ocasiones ha visto el potencial de la educación a distancia. Así, entre otras, la Ley 12/2009, del 10 de julio, de educación de Cataluña, en general su artículo 55 recoge esta importancia, si bien en todo caso mantiene en su apartado $2^{\circ}$ el carácter excepcional de esta enseñanza. Entre otras expresiones, se impone al Departamento de educación programar educación a distancia para compatibilizar con el trabajo para el Bachillerato (art. 61. $4^{\circ}$ ), la formación profesional (art. 62. $9^{\circ}$ ), la enseñanza de idiomas (art. 64. $2^{\circ}$ ) y la educación para adultos (art. $70.1^{\circ}$ ). Asimismo, se dispone la creación y regulación del centro singular para la educación no presencial (Disp. Ad. 28 ). Estos preceptos no han sido impugnados ni, por tanto, declarados inconstitucionales por la STC 51/2019, de 11 de abril sobre esta ley.

En el margen del legislador está considerar como principal la educación presencial en las etapas formativas del menor para el logro de su pleno desarrollo de la personalidad y como se ha visto en la STC 133/2010 sobre escolarización obligatoria parte de la premisa de que el objeto constitucional de la educación -solo- se logra con la interactuación presencial del educando en la escuela. 


\section{IV.- El derecho de acceso a internet y el peligro de la desigualdad en la educación} digital y especialmente en el contexto del coronavirus

Internet es un nuevo paradigma y su acceso ha pasado a ser una necesidad esencial humana que debe estar garantizada como derecho humano y con protección constitucional iusfundamental. Como he tenido ocasión de analizar (COTINO 2009, 2015, 2019), el acceso a internet se ha ido reconociendo paralelamente en el plano internacional de Naciones Unidas, europeo y nacional a modo de un nuevo derecho prestacional. Así en clave internacional se afirmó el "derecho de acceso como acceso universal" a partir del artículo 19 de la Declaración Universal de Derechos Humanos interpretado por Naciones Unidas en la Declaración de Ginebra de 2003 (NACIONES UNIDAS 2003, apartado 3 y números 24 a 28) y la Declaración de Principios de Túnez de 2005 (NACIONES UNIDAS 2005). Después, la importante Declaración conjunta sobre libertad de expresión e internet de 1 de junio de 2011 (Naciones UNIDAS 2011 a) subrayó la "obligación positiva de facilitar el acceso universal a Internet" ( $n^{\circ} 6$ a) y unos mínimos de acción. Se subrayaba la obligación de alfabetización digital, esto es, el deber de "promover la capacidad de todas las personas de efectuar un uso autónomo, independiente y responsable de Internet ("alfabetización digital").” (1. f). Se afirmaba expresamente que "El acceso a Internet también es necesario para asegurar el respeto de otros derechos, como el derecho a la educación" (6. a).

Otras resoluciones internacionales de 2011 (NACIONES UNIDAS 2011 b) y 2012 declararon la protección del acceso vinculado especialmente a la libertad de expresión, destacando también La Corte Suprema de Costa Rica el 30 de julio 2010 que reconoció el derecho fundamental de acceso a internet con obligaciones para el Estado ${ }^{20}$. Y la Declaración del Presidente de los Estados Unidos Barack Obama de 10 de noviembre de $2014^{21}$ reafirmaría el carácter esencial de internet para la economía y la democracia. En 2019, los relatores de libertad de expresión (NACIONES UNIDAS 2019) exhortan a los estados a "Reconocer el derecho al acceso y el uso de internet como un derecho humano y una condición esencial para el ejercicio del derecho a la libertad de expresión." ( $n^{\circ} 2$. a). En buena medida este derecho se ha traducido en la garantía del "acceso universal" esto es, "un conjunto mínimo de servicios, [...] de una calidad determinada y que esté disponible para todo usuario con independencia de su localización geográfica y, a la

20 Decisión del 18-6-2010 (Expediente 10-003560-0007-CO) de la Sala Constitucional de la Corte Suprema de Justicia de Costa Rica.

${ }^{21}$ https://obamawhitehouse.archives.gov/the-press-office/2014/11/10/statement-president-net-neutrality 
vista de las condiciones nacionales específicas, a un precio asequible" (art. 2. J Directiva 2002/21/CE, marco). Todo desarrollado en completa normativa en la UE.

Esta nueva dimensión de un derecho fundamental necesariamente debe conectarse con el derecho a la educación, especialmente cuando sus objetivos deben lograrse a través de internet. Y lo cierto es que los peligros para la igualdad en la educación se dan en muy buena medida con relación a la garantía de acceso y la alfabetización digital.

Para el Relator de educación ONU (2016) "persisten las disparidades en el acceso a las tecnologías digitales, una brecha digital que los países deben superar. La marginación y la exclusión ponen en peligro los principios de equidad y justicia social, que son pilares esenciales de la labor del sistema de las Naciones Unidas en pro de la paz y el desarrollo" ( $\mathrm{n}^{\mathrm{o}}$ 101). Es por ello que con la educación digital se "debe aumentar el acceso, no limitarlo. Debe promover la equidad, no exacerbar las disparidades existentes en la sociedad. Debe eliminar la discriminación, no crear nuevos obstáculos. Debe mejorar la calidad de la educación, no socavarla." Buena parte de su informe monográfico es relativo a las políticas frente a la "brecha digital" en educación (IV): "Disparidades en el acceso a Internet y a la tecnología digital", "Limitaciones de infraestructura" y "Marginación y exclusión”, así como no pocas conclusiones.

En la misma línea, buena parte de las claves de las políticas de educación e internet recomendadas mundialmente se han centrado en evitar la desigualdad. Esencialmente hay que asegurar el acceso asequible para escuelas, docentes y alumnos, así como la alfabetización digital y las otras habilidades necesarias para sacarle provecho.

Así, Internet Society (2017: 2 y ss. ) ha señalado algunas "Consideraciones clave para legisladores", a saber: $\left(1^{\circ}\right)$, sobre los ejes de generar infraestructuras y acceso, acceder en las escuelas a computadoras, tabletas y otros dispositivos TIC para que se integren de manera inteligente en el ambiente educativo para optimizar el uso del aprendizaje facilitado por Internet; $\left(2^{\circ}\right)$ Una visión y política con monitoreo y evaluaciones realistas que incluya todas las etapas del sistema educativo - desde pre-escolar y escuela primaria, pasando por secundario y terciario, hasta el aprendizaje permanente, recapacitación y re-entrenamiento. ( $3^{\circ}$ ) La inclusión en la línea de la Agenda 2030 y tener en cuenta que las TIC pueden exacerbar "desigualdades atrincheradas" si no se toman precauciones. La desigualdad de género en la educación puede ser incluso más fuerte en línea. 
Ya en el marco de la crisis suscitada por el coronavirus, cabe seguir las medidas especiales de equidad e igualdad en razón de la pandemia. Al respecto, De Alonso (2020) ha recordado que el Coronavirus en el ámbito de la educación puede sacar a relucir el panorama lleno de desigualdades que tenemos en la sociedad, y abrir aún más el profundo abismo de desventajas.

Como ha afirmado Harris con motivo de la pandemia (2020), los análisis sobre educación por internet vienen a señalar que los estudiantes desfavorecidos aprenden menos. Ello incluso cuando instituciones y docentes tienen experiencia y capacitación, que no es lo general.

Asimismo y sin duda el contexto familiar, ingresos $y$ otras circunstancias muy vinculadas a la situación económica van a repercutir en la capacidad de lograr los objetivos de la educación a través de internet y bajo las circunstancias a que obliga la pandemia. Por ejemplo, los refuerzos educativos durante los efectos de la pandemia y una vez pasado el pico de la misma van a ser elementos muy necesarios. Y no todos van a tener el mismo acceso a refuerzos en forma de clases particulares o campamentos de verano (HARRIS, 2020).

Sobre igualdad y equidad, más allá de la importancia de asegurar el acceso a comidas nutritivas, Giannini y Grant (2020), indican algunas lecciones aprendidas para evitar desigualdad en razón de la pandemia, en esencia, afrontar la brecha digital (1); capacitar a los maestros para que usen sistemas de gestión de aprendizaje digital (2) y planificar soluciones de aprendizaje inclusivo.

Chang y Yano (2020) especialmente frente a la brecha digital señalan algunos ejemplos de medidas: En Francia, se están haciendo esfuerzos para prestar dispositivos y proporcionar tareas impresas al 5\% de los estudiantes que no tienen acceso a Internet o computadoras; en Portugal, el gobierno sugirió una asociación con los servicios de la oficina de correos para entregar hojas de trabajo; en el estado de Washington, Estados Unidos, no se alienta a las escuelas a proporcionar servicios de aprendizaje en línea a menos que se garantice un acceso equitativo; algunos países mantienen abiertas un pequeño número de escuelas para alojar a los niños que no pueden ser atendidos en el hogar (por ejemplo, Francia , Japón y la República de Corea ); asimismo se planifican incluso visitas periódicas de los maestros a las familias. 
El gobierno de Estados Unidos en el ámbito de educación, además de completa información propiamente sanitaria ${ }^{22}$, lanzó diversos documentos para implementar estrategias para continuar con la educación de los estudiantes y las actividades de apoyo relacionadas: "asegúrese de que continúe la educación”, “de que continúen los programas de alimentación" y "alternativas para proporcionar servicios médicos y sociales esenciales para los estudiantes."

\section{V.- El vaso medio lleno. Las bondades de la transformación digital y una oportunidad para dejar atrás una educación obsoleta del siglo XX}

\section{1.- La necesidad del cambio hacia competencias digitales y el pensamiento computacional}

Frente a otras visiones muy negativas se ha señalado que "Internet tiene un gran potencial para mejorar la calidad de la educación, hacia una gran cantidad de información, conocimiento y recursos educativos, incrementando las oportunidades de aprendizaje dentro y fuera del aula. Facilita a los docentes preparar lecciones y a los alumnos les brinda una amplitud de métodos de enseñanza interactivos, apoyados por Internet. Ello puede permitir atender las necesidades individuales de cada alumno y apoyan el aprendizaje compartido y enmendar las desigualdades. Y también los administradores educativos pueden reducir los costos y mejorar la calidad de escuelas y universidades (INTERNET SOCIETY, 2017, 1).

Mucho más negativo, el Relator para la educación de la ONU (2016) al menos admite que "Las tecnologías digitales están revolucionando la prestación de servicios de educación. Múltiples vías de aprendizaje, como el aprendizaje electrónico, los cursos en línea masivos y abiertos y los recursos educativos abiertos, están contribuyendo a la diversificación de los métodos de aprendizaje $\left(\mathrm{n}^{\mathrm{o}}\right.$ 108) y, cuanto menos por ello merecen ser complementarias de la educación presencial.

La educación debe lograr el pleno desarrollo de la personalidad, y ello hoy día difícilmente puede lograrse sin la plena integración de las TIC en los procesos educativos. Así desde 2004 se recoge en textos internacionales de Naciones Unidas de gran relevancia como la cumbre de Ginebra (NACIONES UNIDAS, 2004: ap. 4º,

\footnotetext{
22 https://www.ed.gov/coronavirus. En concreto, Guía interina para los administradores de escuelas de kínder a 12.o grado y de programas de cuidados infantiles en los EE. UU, marzo 2020, acceso en https://www.cdc.gov/coronavirus/2019-ncov/community/schools-childcare/guidance-for-schools-sp.html
} 
“Creación de capacidad", no 29 a 33 o la Agenda de Túnez (NACIONES UNIDAS 2006: n 90, letras c) d) y k). En la UE, desde la Recomendación de 2006 (UE, 2016: 4º) la "competencia digital" se erige como una de las ocho necesidades y competencias básicas en el marco de la Unión Europea. Esta necesidad no ha hecho más que incrementarse desde 2014 hasta la fecha a través del Marco DigComp, el Marco Común de Competencia Digital Docente, que incluye las siguientes áreas (COMISIÓN EUROPEA 2019; INTEF 2017: 9-10): 1. Información y alfabetización informacional: identificar, localizar, recuperar, almacenar, organizar y analizar la información digital, evaluando su finalidad y relevancia; 2. Comunicación y colaboración: comunicar en entornos digitales, compartir recursos a través de herramientas en línea, conectar y colaborar con otros a través de herramientas digitales, interactuar y participar en comunidades y redes; conciencia intercultural; 3. Creación de contenido digital: crear y editar contenidos nuevos (textos, imágenes, videos...), integrar y reelaborar conocimientos y contenidos previos, realizar producciones artísticas, contenidos multimedia y programación informática, saber aplicar los derechos de propiedad intelectual y las licencias de uso; 4. Seguridad: protección personal, protección de datos, protección de la identidad digital, uso de seguridad, uso seguro y sostenible; 5. Resolución de problemas: identificar necesidades y recursos digitales, tomar decisiones a la hora de elegir la herramienta digital apropiada, acorde a la finalidad o necesidad, resolver problemas conceptuales a través de medios digitales, resolver problemas técnicos, uso creativo de la tecnología, actualizar la competencia propia y la de otros (INTEF, 2017).

La Internet Society (2017: 2 y ss. $\left.4^{\circ}\right)$ no ha dudado en reconocer que "el éxito en la era digital requiere habilidades digitales. La utilidad de las TIC para la creación de capacidad de los educandos de cara a futuros empleos, emprender negocios, llevar vidas plenas y proveer para ellos mismos y sus familias. Para ello, los docentes deben adquirir nuevas habilidades para utilizar eficazmente los recursos de Internet. Finalmente, generar y filtrar contenidos y herramientas de calidad, así como adecuarse a los dispositivos de uso generalizado."

Nuestra legislación, especialmente en la Ley Orgánica 2/2006, de 3 de mayo, de Educación (LOE), ha recibido transversalmente esta competencia digital en todas las etapas educativas. Así se observa ya en el segundo ciclo de educación infantil (art. 14), y de forma creciente en Primaria (art. 17 y 19. $2^{\circ}$ ), en la ESO (arts. 23 e), $24.7^{\circ}$ y $25.5^{\circ}$ 
LOE), en Bachillerato y en la formación de personas adultas en la formación a distancia (art. 69 LOE). También en la LOE se recoge la necesidad de formación del profesorado en TIC (arts. 102. $3^{\circ}$ y 157 LOE) y de dotación de medios de los centros (art. 112. $2^{\circ}$ LOE).

En buena medida, las exigencias constitucionales de contextualización del derecho a la educación a la sociedad quedan colmadas con la regulación. Sin embargo, a lo que hay que estar es a los medios y los resultados.

Pero lo cierto es que pese a las continuas referencias a la importancia de la educación y las competencias digitales, la percepción generalizada tanto en España como en muchos países es que no se avanzaba ni mucho menos lo que se debía. Desde la OCDE afirma Sikka (2016: 17) que las aulas de hoy a menudo funcionan de la misma manera que cuando los agricultores componían la mayoría de nuestras sociedades. Y también desde la OCDE Partovi (2019) apunta que en la mayoría de las escuelas se ven maestros enseñando exactamente la misma materia que en 1918: lectura, escritura, matemáticas, ciencias, historia e idiomas extranjeros.

Así las cosas se insiste en que el intensivo uso de TIC puede ser un revulsivo para intensificar también las competencias en pensamiento computacional, diseño de interfaz, análisis de datos, aprendizaje automático, ciberseguridad, redes y robótica que fomenta la creatividad, la resolución de problemas, la ética y la colaboración. De hecho en 2021 la evaluación matemática PISA incorporará preguntas que evalúen el pensamiento computacional: el enfoque lógico o de resolución de problemas que sirve como la columna vertebral conceptual de las tecnologías digitales ${ }^{23}$.

La resistencia al cambio y a la innovación en la educación es fuerte. Como ya decía Álvarez en 2001, se resiste quien no está acostumbrado a trabajar en equipo, quien se siente amenazado por los cambios, quien se conforma con la situación que le viene dada, se resiste quien es incapaz de cambiar sus hábitos y seguridad, quien ve en peligro privilegios y derechos adquiridos. Y también quien animado por el Proyecto no encuentra ni medios ni apoyos quien desconfía de tanta promesa cuando comprueba que las condiciones no se cumplen (ÁLVAREZ MÉNDEZ, 2001).

\footnotetext{
${ }^{23} \mathrm{http}$ ://www.oecd.org/pisa/pisaproducts/pisa-2021-mathematics-framework-draft.pdf
} 
Pues bien, la crisis de la pandemia nos obliga a adecuarnos e innovar. Se ha afirmado con razón que "No se debe olvidar jamás que lo importante es el cambio, no los metros cuadrados de aulas, ni las horas de enseñanza, ni el detalle de los programas, ni tampoco los créditos, sino las personas y la relación entre las mismas. Y que toda evolución en este terreno demanda tiempo". (MARGALEF y otros, 2006: 17).

Todo parece indicar que algo tan biológico como el coronavirus ha sido el empujón que nos faltaba para el salto a la transformación digital en la educación.

\section{2.- La maldita pandemia, una oportunidad}

Unicef (2020) con la generalización de la pandemia ha señalado que "la educación es un salvavidas para los niños y las niñas." La educación permite a los menores generar rutinas escolares que les proporcionan la estabilidad y el orden que les ayudan a afrontar la incertidumbre. Se ha señalado que puede ser fuente de aprendizajes valiosos sobre salud y prevención de riesgos y, con apoyo la familia puede ayudar a canalizar las emociones.

Especialmente implica un salto para el avance de la educación digital. Desde el World Economic Forum, Luthra y Mackenzie (2020) afirman que la experiencia "puede enseñarnos sobre cómo debe cambiar la educación para poder preparar mejor a nuestros jóvenes". Entre otras, la pandemia ha supuesto el obligado y repentino desbloqueo de tecnología para impartir educación. La misma situación de aislamiento puede ser un revulsivo para valorar la importancia de la interacción social cara a cara para el futuro. Asimismo, señalan que es una oportunidad de comprender la interrelación e interconexión mundial; ha de llevar a redefinir el papel del educador como facilitador del desarrollo de los jóvenes, no como un transmisor de conocimiento que tienen a un clic. La situación implica necesaria adaptabilidad, va a llevar a enseñar habilidades para la vida necesarias para el futuro como la creatividad, la comunicación y la colaboración, junto con la empatía y la inteligencia emocional. De Alonso (2020) apunta también las aportaciones de las Nuevas Tecnologías al aprendizaje dialógico, interactivo, colaborativo y social, elementos muy necesarios hoy en día. 


\section{VI.- Los deberes del Estado y las familias para la realización del derecho a la educación esencialmente a través de internet ante el coronavirus}

Unicef (2020) ha señalado con acierto que la urgencia de la situación de la pandemia del coronavirus ha obligado a dar prioridad a la vida, integridad y salud frente al derecho a la educación. No obstante, este derecho humano y fundamental debe hacerse efectivo hasta al máximo posible también en estas circunstancias. Y aunque no solo, internet es el salvavidas del derecho a la educación. Ello es así pese a las múltiples dificultades y barreras que implica.

Aunque puede tratarse de una obviedad, desde la Relatoría de educación ONU (2016) se ha subrayado que es "importante garantizar que cuando se utilicen las tecnologías de la información y las comunicaciones se preserven los principios y normas del derecho a la educación" ( $\left.n^{\circ} 44\right)$; "La utilización de las tecnologías digitales en la educación deben ajustarse plenamente al derecho a la educación" ( $\left.\mathrm{n}^{\mathrm{0}} 100\right)$ e incluso se afirma "Un enfoque de las tecnologías digitales basado en los derechos" ( $\left.{ }^{\circ} 119\right)$. Para ello, deben darse "disposiciones prescriptivas, prohibitivas y punitivas sobre la utilización de la tecnología digital en la educación con miras a garantizar que el derecho a la educación esté debidamente protegido" ( $\mathrm{n}^{\circ}$ 104), para ello, generarse "mecanismos de garantía de calidad" ( $\left.\mathrm{n}^{\mathrm{o}} 102\right)$ y rendición de cuentas $\left(\mathrm{n}^{\circ} 103\right)$.

Procede recordar el contenido del derecho a la educación y las obligaciones que comporta para el Estado y para los padres y madres a los efectos de ver la proyección de este derecho en general en la educación digital y, especialmente, ante la pandemia.

\section{1.- Las obligaciones del Estado en razón del derecho a la educación y su potencial proyección para el ámbito digital}

En otros lugares he tenido ocasión de analizar exhaustivamente el derecho a la educación (COTINO 2011, 2012). El "derecho a la educación” describe todo el sistema educativo constitucional (STC 86/1985, FJ 3º), esto es, la ordenación de la prestación de las enseñanzas regladas (art. 3. $1^{\circ} \mathrm{LOE}$ ). Y en razón de este derecho fundamental se configura un servicio público, se trata además de un "servicio esencial de la comunidad" (Preámbulo de la LOE y DOMÍNGUEZ-BERRUETA y SENDÍN 2005, 29). Es asimismo un servicio público objetivo con independencia de la naturaleza del prestatario: así, "La prestación del servicio público de la educación se realizará, a través 
de los centros públicos y privados concertados" (art. 108. 4 LOE) o de universidades públicas o privadas (art. 3 Ley Orgánica 6/2001, de 21 de diciembre, de Universidades).

En todo caso se trata de un derecho subjetivo, fundamental y prestacional, un "derecho de carácter social" (Preámbulo de la LOGSE). Se ha afirmado que el derecho a la educación en su dimensión subjetiva "implica el correlativo a disponer de la plaza escolar en un centro educativo, como soporte físico e instrumental que permite recibir la enseñanza adecuada" (STS de 22 diciembre 2003, con cita de STS 8 de julio de 1986). Del "derecho a la educación" se deriva el correlato de la obligación estatal a una "acción positiva fáctica". Siguiendo el claro esquema internacional del artículo 2 del Pacto Internacional de Derechos Económicos, Sociales y Culturales, hecho en Nueva York el 19 de diciembre de 1966 (PIDESC), se ha de "lograr progresivamente [...] la plena efectividad de los derechos" "hasta el máximo de los recursos de que disponga".

El Comité de Derechos Económicos, Sociales y Culturales (DESC) (1999) hizo una interpretación autorizada en su Observación General no 13 del contenido del derecho a la educación PIDESC: el acceso a las instituciones y programas de enseñanza públicos sin discriminación; la implantación prioritaria de la enseñanza primaria obligatoria y gratuita para todos. Igualmente se estima imprescindible la adopción de "medidas deliberadas, concretas y orientadas" hacia la implantación gradual de la enseñanza secundaria, superior y fundamental. Se considera esencial que los planes de estudio no sean incompatibles con el objeto declarado de la educación, con sistemas eficaces de control. También se reputa un contenido mínimo la libre elección de la educación sin la intervención del Estado ni de terceros, si bien las enseñanzas privadas deben cumplir mínimos y finalmente, la garantía de la libertad académica del cuerpo docente.

Sin perjuicio de ese contenido prestacional básico, el derecho a la educación implica una serie de derechos concretos y las consiguientes obligaciones principalmente de los poderes. Y éstos son objetivos que deben hacerse realidad en general en la educación digital y en particular en razón de la pandemia. Se trata de los derechos a la escolarización en los diversos niveles, a recibir una enseñanza asequible (presupuesto suficiente, escuelas y maestros necesarios, infraestructura y dotaciones pertinentes). El derecho a una enseñanza de las cuatro A: accesible (el derecho a elegir centro, el derecho de acceso y admisión, la eliminación de barreras materiales y asequibilidad efectiva a las enseñanzas, acceso a sistema de becas y ayudas, gratuidad); aceptable (educación de calidad, ajustada al objeto constitucional de la educación y evitación del 
fracaso escolar) y adaptable. Asimismo los derechos a no ser discriminado, a ser evaluado objetivamente y con garantías, a permanecer y progresar, al reconocimiento oficial de los estudios realizados y los títulos correspondientes, a ser informado y orientado y a participar en la planificación de la enseñanza y en la gestión de los centros subvencionados con dinero público. Estos objetivos pueden medirse con indicadores y, pese a que los poderes públicos elijan las diferentes vías, jurídicamente es controlable el logro de los mismos.

\section{2.- Las obligaciones prestacionales del derecho a la educación deben proyectarse a la educación digital}

El derecho a la educación como derecho prestacional es terreno abonado a la actividad normativa legal y a la colaboración reglamentaria. Más allá de la propia acción normativa implica acciones y recursos. Así, además de la gratuidad de la educación básica, el derecho a la educación incluye servicios escolares de transporte, comedor o internado en los casos de zonas rurales donde sea necesaria la escolarización en municipios próximos (art. 82. $3^{\circ} \mathrm{LOE}$ ). Asimismo, hay un derecho fundamental de acceso a las ayudas y becas y para ello, entre otras cosas, se exige "implantar un sistema adecuado de becas" (art. 13. $2^{\circ}$ PIDESC). La STC 188/2001, de 20 septiembre FJ $5^{\circ}$ es muestra del proceso de fundamentalización del derecho a obtener becas y ayudas, pues como señala la STC 236/2007, de 7 noviembre FJ $8^{\circ}$ las becas son "un elemento central para la efectividad de tal derecho".

La dotación de medios materiales y humanos es imprescindible para la efectividad del derecho a la educación con una calidad adecuada que permita la igualdad de oportunidades. La LOE exige dotar de medios a los centros con medios materiales, humanos, incorporando tecnologías, programas de refuerzo, etc. (arts. $112.1^{\circ}$ y $3^{\circ}, 122$. $1^{\circ}$ y el $\left.157 \mathrm{LOE}\right)$. La normativa es muy prolija.

El incumplimiento de estas exigencias es motivo suficiente para denegar la autorización de un centro privado. Respecto de los medios vinculados a las nuevas tecnologías, la legislación señala la necesidad de formación del profesorado en TICs (arts. 102. $3^{\circ} \mathrm{y}$ 157 LOE) y de dotación de medios de los centros (art. 112. $2^{\circ}$ LOE). El Tribunal Constitucional ya afirmó que "no puede desconocerse la relevancia que la utilización de medios informáticos tiene hoy en el ámbito educativo" (STC 140/2002, de 3 junio, FJ $\left.5^{\circ}\right)$. 
La negación de algunos medios y prestaciones adicionales puede hacer ilusorio hablar de derecho a la educación. Si el derecho fundamental no puede ser ejercitado sin dichos medios, la obligación de prestación de los medios pasa a integrarse en el contenido subjetivo del derecho fundamental (ALÁEZ CORRAL, 2003, 134).

Bien es cierto que, pese a que se trate de un derecho fundamental de los que cuentan con máxima protección, los tribunales por lo general parten de una desfundamentalización. En general, entienden que la regulación de los requisitos, condiciones, organización y procedimiento de la prestación del servicio público educativo son elementos que aun siendo necesarios para la eficacia del derecho, no constituyen la "esencia" del mismo, sino cuestiones de "mera legalidad". Así, entre otras y como botón de muestra la STS de 13 octubre 1995: "Siendo el derecho a la educación un derecho de prestación, y regulada ésta por normas infraconstitucionales, que establecen requisitos materiales y formales para la impartición de la misma, las cuestiones referentes al cumplimiento de los requisitos infraconstitucionales, o al modo de producción de los actos administrativos, y a la invalidez de éstos, tienen en principio el significado de cuestiones de legalidad ordinaria, sin que afecten al derecho fundamental, como la parte da por sentado.”

No obstante, como sostuve hace años $(2011,2012)$, la lesión del derecho a la educación por la falta de medios para su realización es un juicio asequible jurídica y judicialmente. En este sentido, frente a la referida tolerancia judicial destacó la STS de 9 de mayo de 2011 que estimó lesión del derecho fundamental por la inactividad de la Generalitat Valenciana por la falta del material adecuado y sin personal educativo con la formación específica para atender a estos alumnos. El hecho de que se tratara de niños autistas fue el elemento decisivo. A nivel infraconstitucional, los libros de texto gratuitos han sido reconocidos como "derecho" en el artículo 21. $5^{\circ}$ del Estatuto de autonomía de Andalucía, con desarrollo legislativo, así en leyes como la Ley Foral 6/2008, de 25 de marzo de Navarra o en el artículo 130 Ley 7/2010, de 20 de julio, Ley de Educación de Castilla-La Mancha.

Los docentes son los prestadores reales del servicio educativo, los que ejecutan la actividad material en que consiste la prestación. Los docentes y los centros están en la primera línea del ejercicio del derecho a la educación y del logro efectivo del objeto de la educación 
Pues bien, sobre esta base hay que proyectar las exigencias de satisfacer en la medida de lo posible del derecho a la educación cuando deba ser a través de medios digitales. Los poderes públicos y la comunidad educativa tienen que hacer los máximos esfuerzos. La falta de acciones y medios adecuados suficientes para lograr los objetivos del derecho a la educación implicará una lesión de este derecho. Y ello vale tanto en general para la educación a distancia, como en particular para garantizar la educación en razón del coronavirus.

\section{3.- Un recordatorio de los deberes de los padres en la educación, también la digital en razón de las circunstancias}

Sin perjuicio de los deberes de los poderes públicos y del protagonismo de centros y docentes, los padres u otras personas responsables de la educación del menor son los "primeros educadores", como afirmase la relatora del derecho a la educación en 2004 (NACIONES UNIDAS- TOMASEVSKI, 2004) y, al tiempo, son los "primeros responsables" (art. 5. $2^{\circ}$ LODE) de que sus hijos reciban la educación a la que tienen derecho. La STEDH de 7 febrero 2006 (asunto D. H. y otros contra la República Checa, $n^{\circ}$ 51) recuerda que los padres "tienen el deber natural de asegurar la educación de sus hijos", así como informarse de las posibilidades que ofrece el Estado y en su caso impugnar decisiones de las autoridades en bien de su hijo. Así pues, los padres son los primeros destinatarios de la obligación y al Estado le corresponde una función supervisora y aseguradora, como ratifica el artículo 39. $3^{\circ} \mathrm{CE}$ ("Los padres deben prestar asistencia de todo orden a los hijos"). De este modo se explica que la patria potestad "se ejercerá siempre en beneficio de los hijos" "y comprende" el deber de "educarlos y procurarles una formación integral" (art. 154 Código Civil).

Como señala el TEDH, la negación del derecho a la educación de los hijos causada por los padres puede llegar a acarrear la pérdida de la patria potestad, es decir, llegar a justificar una injerencia tan grave en el derecho a la vida privada y familiar (STEDH, Asunto Olsson de 24 de marzo de 1988). Cabe recordar que el artículo 5. $2^{\circ}$ LODE señala que corresponde a los padres, entre otras cosas "a) Adoptar las medidas necesarias, o solicitar la ayuda correspondiente en caso de dificultad, para que sus hijos o pupilos cursen las enseñanzas obligatorias y asistan regularmente a clase; b) Proporcionar, en la medida de sus disponibilidades, los recursos y las condiciones necesarias para el progreso escolar. c) Estimularles para que lleven a cabo las actividades de estudio que se les encomienden. d) Participar de manera activa en las 
actividades que se establezcan en virtud de los compromisos educativos que los centros establezcan con las familias, para mejorar el rendimiento de sus hijos." Se trata de una responsabilidad que va más allá de la exigencia propiamente jurídica, pero que resulta necesario recordar ante una situación excepcional como la relativa a la pandemia. Y obviamente, ante un contexto de necesidad es preciso recordar deberes y responsabilidades también de padres y tutores.

\section{VII.- A modo de conclusión y la necesidad de estrategias para una enseñanza online inesperada}

Que la educación presencial es necesaria e incluso superior a la digital, no hay duda. Que la crisis nos brinda la oportunidad de mejorar mucho la educación gracias a la educación digital, también.

Las consecuencias del coronavirus son y serán indudablemente odiosas para la humanidad a corto y medio plazo en todos los órdenes. Y la educación y especialmente los niños se van a resentir. No obstante, ha tenido que ser la biología la que empuje al ser humano a la transformación digital y con ella a la educación a través de las TIC y, hay que esperar, hacia las competencias digitales. El "tsunami" digital en la educación se ha percibido muy negativamente hasta la fecha. Sin embargo, es un salvavidas en una situación como la pandemia. Este salvavidas, a corto plazo, puede llevar a garantizar unos mínimos de los objetivos que garantiza el derecho a la educación. Y para ello es necesario una acción positiva fáctica y jurídica desde los poderes públicos y toda la comunidad educativa. Tenemos todos una oportunidad histórica de que las secuelas se minimicen en la enseñanza si jugamos bien todas las bazas. Pero es más, el maldito coronavirus nos obliga a todos a asumir el cambio de siglo y a exprimir todas las posibilidades positivas que brinda la educación digital. Hay que mitigar todos los efectos del vaso medio vacío de la educación digital, máxime sin la previsión y urgencia hay que esforzarse y mucho para garantizar el derecho a la educación a través de la enseñanza online y especialmente en estas circunstancias. Ahora bien, también, cuando normalicemos la excepcionalidad y con cierta perspectiva, esta situación quizá nos despierte de un letargo que nos llevaba a hablar mucho de la educación digital, pero ésta se hacía esperar como el Vuelva Ud. mañana de Larra.

La escasa práctica en los últimos años nos ha abocado a tener que normalizar la educación online de manera urgente. Además de las acciones que se han ido 
describiendo, entre otras las relativas al acceso efectivo, plataformas y contenidos educativos se ha subrayado la necesidad de planificación y estrategias para la docencia en línea. En esta dirección cabe recordar el "Decálogo para una docencia en línea inesperada" desde la experiencia de la UOC (EDUL@AB, 2020). De manera sintética, cabe recordar la necesidad de: 1 . Seleccionar el sistema y las herramientas de trabajo más adecuadas para cada objetivo y facilitar tutoriales para que a todos, familias incluidas, les resulte fácil su aplicación; 2. Organizar y preparar a los alumnos; 3. Dedicar tiempo al diseño (re-diseño) del curso y re-diseñar algunas actividades concretas; 4. Elaborar un conjunto de actividades, y acompañadlas de un conjunto de recursos didácticos que ayuden a los estudiantes a resolverlas; 5 . Asociar un conjunto de recursos a las actividades elaborados por iniciativa propia y a partir de recursos de calidad en la red. Es necesario que las imágenes y los sonidos las pueda interpretar un lector de texto, para aquellas personas que no ven o no oyen; 6. Crear dinámicas de interacción activa en el entorno virtual; 7. Explicar el modelo de evaluación y tener en cuenta la evaluación continua como una herramienta que facilita el seguimiento; 8. Generar presencia social, los alumnos no presenciales corren el riesgo de sentirse solos. Hacedles sentir que forman parte de una comunidad donde todos tienen el mismo objetivo; 9. Desarrollar el espíritu crítico de los estudiantes respecto a la tecnología; 10. Aprovechar para trabajar colaborativamente con los compañeros y compañeras docentes más cercanas.

\section{Bibliografía}

ALÁEZ CORRAL, BENITO (2003). Minoría de edad y derechos fundamentales, Tecnos, Madrid, 2003. Acceso completo en https://www.unioviedo.es/constitucional/miemb/alaez/menor.pdf

ÁLVAREZ MÉNDEZ, J. M. (2001). Entender la didáctica, entender el curriculum. Miño y Dávila. Madrid.

CHANG, GWANG-CHOL Y YANO, SATOKO. (2020). "How are countries addressing the Covid-19 challenges in education?" UNESCO's Section of Education Policy A snapshot of policy measures, 24.3.2020. Acceso en https://gemreportunesco.wordpress.com/2020/03/24/how-are-countries-addressing-thecovid-19-challenges-in-education-a-snapshot-of-policy-measures/

COMISIÓN EUROPEA (2019). DigComp: The European Digital Competence Framework

https://ec.europa.eu/social/main.jsp?catId=738\&langId=es\&pubId=8203\&furtherPubs= yes 
COMITÉ DE DERECHOS ECONÓMICOS, SOCIALES Y CULTURALES NACIONES UNIDAS $\left(1999\right.$ b). Observación General $n .^{\circ} 13$. El derecho a la educación (art. 13 del PIDESC). Doc. E/C.12/1999/10, de 8 de diciembre de 1999.

\section{COTINO HUESO, LORENZO:}

- "Derecho a la educación" (2011). en ESCOBAR ROCA, Guillermo (dir.), Derechos sociales y tutela antidiscriminatoria, Thomson-Aranzadi, Cizur Menor. Acceso en https://dialnet.unirioja.es/servlet/libro?codigo=577352\&orden=0\&info=open_link_libro

- El derecho a la educación como derecho fundamental. Especial atención a su dimensión social prestacional, (2012). Centro de Estudios Políticos y Constitucionales, acceso completo http://documentostics.com/component/option,com_docman/task,doc_download/gid,153 $\underline{4 /}$

- "Los derechos fundamentales en tiempos del Coronavirus. Régimen general y garantías y especial atención a las restricciones de excepcionalidad ordinaria" (2020 a) en El Cronista, IUSTEL, monográfico Coronavirus... y otros problemas, marzo-abril 2020, pp. 88-101. Acceso particular en Academia, todo el número en http://www.elcronista.es/El-Cronista-n\%C3\%BAmero-86-87-Coronavirus.pdf-.

-"Confinamientos, libertad de circulación y personal, prohibición de reuniones y actividades y otras restricciones de derechos por la pandemia del coronavirus", Diario La Ley 2020.

- "Online-offline. Las garantías para el acceso a internet y para la desconexión, bloqueo, filtrado y otras restricciones de la red y sus contenidos" (2020 c), de próxima publicación.

DE ALONSO PAZ, ALBANO, "Coronavirus", IDED21, https://ined21.com/educar-enlos-tiempos-del-virus/

DOMÍNGUEZ-BERRUETA DE JUAN, Miguel Ángel y SENDÍN GARCÍA, Miguel Ángel (2005) Derecho y educación: régimen jurídico de la educación,: Ediciones Universidad de Salamanca, Salamanca.

EDUL@AB, Decálogo para una docencia en línea inesperada. Decálogo para estudiar en linea en tiempos de coronavirus, Edul@ab- UOC. 16.3.2020 http://edulab.uoc.edu/es/2020/03/16/decalogo-para-docencia-linea-inesperada-2/

GIANNINI, STEFANIA Y GRANT LEWIS, SUZANNE. (2020). Three ways to plan for equity during the coronavirus school closures, 25.3.2020, UNESCO, (UNESCO Assistant Director-General for Education and, -UNESCO-IIEP Director). Acceso en https://gemreportunesco.wordpress.com/2020/03/25/three-ways-to-plan-for-equityduring-the-coronavirus-school-closures/ 
GOODMAN, JOSHUA, "Flaking Out: Student Absences and Snow Days as Disruptions of Instructional Time" (2014) NBER Working Paper No. 20221 junio 2014 NBER Papers on Economics of Education Program, acceso en https://www.nber.org/papers/w20221

GUIMÓN, PABLO (2019). "Los gurús digitales crían a sus hijos sin pantallas". El País, Crecer conectados, 20.3.2019. Acceso en https://elpais.com/sociedad/2019/03/20/actualidad/1553105010_527764.html

HARRIS, DOUGLAS N. (2020). Using federal stimulus to get schools through the coronavirus crisis: The case for summer school and summer teacher pay. Brookings Institution, 11 de marzo. Acceso en https://www.brookings.edu/blog/brown-centerchalkboard/2020/03/11/using-federal-stimulus-to-get-schools-through-the-coronaviruscrisis-the-case-for-summer-school-and-summer-teacher-pay/

INTEF (2017), Marco Común de Competencia Digital Docente, INTEF, ministerio de Educación, acceso en http://educalab.es/documents/10180/12809/Marco+competencia+digital+docente+2017/ afb07987-1ad6-4b2d-bdc8-58e9faeeccea

INTERNET SOCIETY (2017). Acceso a Internet y educación: Consideraciones clave para legisladores, Internet Society, noviembre, acceso en https://www.internetsociety.org/wp-content/uploads/2017/11/Internet-AccessEducation_ES.pdf

JAUME DAVID Y WILLÉN, ALEXANDER, "The Long-Run Effects of Teacher Strikes: Evidence from Argentina", Journal of Labor Economics 2019 37:4, 1097-1139. Acceso completo en http://barrett.dyson.cornell.edu/NEUDC/paper_179.pdf

LUTHRA POORNIMA Y SANDY MACKENZIE (2020). 4 ways COVID-19 could change how we educate future generations, WEF, Education and Skills, 30 marzo, acceso en https://www.weforum.org/agenda/2020/03/4-ways-covid-19-educationfuture-generations/

MARCELO CABROL, "Las escuelas y el coronavirus, tres desafíos urgentes y una transformación necesaria", en Enfoque Educación, BID, marzo 16, 2020, https://blogs.iadb.org/educacion/es/escuelasycoronavirus/

MARGALEF GARCÍA, LEONOR; ARENAS MARTIJA, ANDONI (2006) “¿Qué entendemos por innovación educativa? A próposito del desarrollo curricular". Perspectiva Educacional, Formación de Profesores, núm. 47, 2006, pp. 13-31 Pontificia Universidad Católica de Valparaíso.

NACIONES UNIDAS (2003). Declaración de Ginebra, Cumbre Mundial sobre la Sociedad de la Información. 
NACIONES UNIDAS (2004) Declaración de Principios de Ginebra, Cumbre Mundial de la Sociedad de la Información "Construir la sociedad de la información: un desafio global para el nuevo milenio", Documento WSIS-03/GENEVA/4-S12 de mayo de 2004.

NACIONES UNIDAS (2005). Declaración de Principios de la Segunda Fase de Cumbre de Ginebra, celebrada en Túnez en Noviembre de 2005, conocida como Compromiso de Túnez, que incluye un Plan de Acción sobre la Sociedad de la Información.

NACIONES UNIDAS (2006). Agenda de Túnez para la sociedad de la información, de 28 de junio de 2006, Documento WSIS-05/TUNIS/DOC/6(Rev.1)-S

NACIONES UNIDAS (2012). Resolución del Consejo de Derechos Humanos ONU de 5 de julio de 2012 "Promoción, protección y disfrute de los derechos humanos en Internet" http://ap.ohchr.org/documents/S/HRC/d_res_dec/A_HRC_20_L13.pdf

NACIONES UNIDAS (2019), Declaración Conjunta del Vigésimo Aniversario: Desafios para la Libertad de Expresión en la Próxima Década, 10 Julio 2019, https://www.refworld.org.es/docid/5d30a7d84.html

NACIONES UNIDAS- RELATOR ESPECIAL LIBERTAD DE EXPRESIÓN (2011 b). Informe a la Asamblea de Naciones Unidas de 16 de mayo de 2011 sobre la promoción y protección del derecho a la libertad de opinión y de expresión, Frank La Rue http://www.un.org/ga/search/view_doc.asp?symbol=A/HRC/17/27\&Lang=S

NACIONES UNIDAS- TOMASEVSKI, Katarina (2004) Informe sobre el derecho a la educación, de 25 de enero de 2004, (Relatora de educación para el Consejo de Naciones Unidas, Katarina Tomasevski), Documento E/CN.4/2004/45.

NELLIE BOWLES (2019) "Los tecnólogos de Silicon Valley no quieren que sus hijos usen los dispositivos que ellos fabrican". The New York Times, Tecnología, 27 de octubre de 2018, https://www.nytimes.com/es/2018/10/27/espanol/cultura/hijosdispositivos-moviles-silicon-valley.html

PARTOVI, HADI (2019). Why schools should teach the curriculum of the future, not the past, en OECD Education and Skills Today, https://www.weforum.org/agenda/2018/09/why-schools-should-teach-the-curriculumof-the-future-not-the-past/

RELATOR ESPECIAL EDUCACIÓN DE LA ONU (2016), Informe de 2015 del Relator Especial sobre el derecho a la educación (dedicado a derecho a la educación en la era digital ) Consejo de Derechos Humanos (A/HRC/32/37), 6.4.2016, acceso en español http://daccess-ods.un.org/access.nsf/Get?Open\&DS=A/HRC/32/37\&Lang=S 
RELATORES DE LIBERTAD DE EXPRESIÓN (2011). Declaración conjunta sobre libertad de expresión e internet, acceso en http://www.oas.org/es/cidh/expresion/showarticle.asp?artID=849\&lID=2

SANZ, ISMAEL, "Efectos de la crisis del coronavirus sobre la educación", en Magisterio, 19.3.2020, https://www.magisnet.com/2020/03/efectos-de-la-crisis-delcoronavirus-sobre-la-educacion/

SIKKA VISHAL (2016). "What kind of education do we need in the future?", en OECD Education and Skills Today, https://www.weforum.org/agenda/2016/01/amplifying-our-human-potential-a-newcontext-for-the-fourth-industrial-revolution/

UE PARLAMENTO Y CONSEJO DE LA UNIÓN EUROPEA (2006) Recomendación 2006/962/CE del Parlamento Europeo y del Consejo, de 18 de diciembre de 2006, sobre las competencias clave para el aprendizaje permanente.

UNESCO (2020 a), COVID-19 Educational Disruption and Response, UNESCO, https://en.unesco.org/themes/education-emergencies/coronavirus-school-closures

UNESCO (2020 b). Distance learning solutions, UNESCO, Marzo 2020, Acceso en https://en.unesco.org/themes/education-emergencies/coronavirus-schoolclosures/solutions

UNICEF (2020). El nuevo coronavirus y el derecho a la educación, 5.3.2020, https://www.unicef.es/educa/blog/nuevo-coronavirus-derecho-educacion 